\title{
Health-related quality of life and treatment
} satisfaction in patients with gout: results from a cross-sectional study in a managed care setting

\author{
This article was published in the following Dove Press journal: \\ Patient Preference and Adherence \\ 9 July 2015 \\ Number of times this article has been viewed
}

\section{Puja P Khanna' \\ Aki Shiozawa ${ }^{2}$ \\ Valery Walker ${ }^{3}$ \\ Tim Bancroft ${ }^{3}$ \\ Breanna Essoi ${ }^{3}$ \\ Kasem S Akhras ${ }^{4}$ \\ Dinesh Khanna'}

'Department of Internal Medicine, University of Michigan, Ann Arbor, MI, USA; ${ }^{2}$ Global Outcome Research, Takeda Pharmaceuticals International, Inc., Deerfield, IL, USA; ${ }^{3}$ Health

Economics and Outcomes Research, Optum, Eden Prairie, MN, USA;

${ }^{4}$ Novartis Pharmacy Services AG, Dubai, United Arab Emirates
Correspondence: Aki Shiozawa Global Outcome Research, Takeda Pharmaceuticals International, Inc., One Takeda Parkway, Deerfield, IL 600I5, USA

$\mathrm{Tel}+\mathrm{I} 224554716$ I

Fax + I 2245547983

Email aki.shiozawa@takeda.com
Background: Patient satisfaction with treatment directly impacts adherence to medication. Objective: The objective was to assess and compare treatment satisfaction with the Treatment Satisfaction Questionnaire for Medication (TSQM), gout-specific health-related quality of life (HRQoL) with the Gout Impact Scale (GIS), and generic HRQoL with the SF-12v2 ${ }^{\circledR}$ Health Survey (SF-12) in patients with gout in a real-world practice setting.

Methods: This cross-sectional mail survey included gout patients enrolled in a large commercial health plan in the US. Patients were $\geq 18$ years with self-reported gout diagnosis, who filled $\geq 1$ prescription for febuxostat during April 26, 2012 to July 26, 2012 and were not taking any other urate-lowering therapies. The survey included the TSQM version II (TSQM vII, score 0-100, higher scores indicate better satisfaction), GIS (score 0-100, higher scores indicate worse condition), and SF-12 (physical component summary and mental component summary). Patients were stratified by self-report of currently experiencing a gout attack or not to assess the discriminant ability of the questionnaires.

Results: A total of 257 patients were included in the analysis (mean age, 54.9 years; $87 \%$ male). Patients with current gout attack $(n=29,11 \%)$ had worse scores than those without gout attack on most instrument scales. Mean differences between current attack and no current attack for the TSQM domains were: -20.6 , effectiveness; -10.6 , side effects; -12.1 , global satisfaction (all $P<0.05$ ); and -6.1 , convenience (NS). For the GIS, mean differences were: 30.5 , gout overall concern; 14.6, gout medication side effects; 22.7, unmet gout treatment needs; 11.5, gout concern during attack (all $P<0.05$ ); and 7.9, well-being during attack (NS). Mean difference in SF-12 was -6.6 for physical component summary $(P<0.05)$ and -2.9 for mental component summary (NS). Correlations between several TSQM and GIS scales were moderate.

Conclusion: The TSQM and GIS were complementary in evaluating the impact of gout flare on treatment satisfaction and HRQoL. Correlations between the two instruments supported the relationship between treatment satisfaction and HRQoL.

Keywords: febuxostat, gout, gouty arthritis, Treatment Satisfaction Questionnaire for Medicine, Gout Impact Scale, SF-12

\section{Introduction}

Gout is an inflammatory arthritis caused by deposition of monosodium uric acid crystals in the joints and surrounding tissues (tophi) as a result of excess uric acid burden. ${ }^{1,2}$ Acute gout attacks manifest as painful, swollen joints; recurrent flares lead to chronic tophaceous gout, resulting in joint damage and disability. ${ }^{2,3}$ Gout and gout flares are associated with reduced health-related quality of life (HRQoL) $)^{4-7}$ and higher 
health care costs. ${ }^{8,9}$ In the US, the estimated prevalence of gout has increased sharply over the last few decades, from $2.9 \%$ of adults in $1988-1994$ to $3.9 \%$, or 8.3 million adults, in 2007-2008. ${ }^{10}$

The American College of Rheumatology (ACR) and European League Against Rheumatism (EULAR) guidelines for the pharmacologic management of acute gout attacks include oral colchicine, non-steroidal anti-inflammatory drugs, and corticosteroids. ${ }^{11,12}$ For the management of chronic gout, ACR guidelines recommend long-term urate-lowering therapy (ULT) for patients diagnosed with gouty arthritis and meeting one of four criteria, including frequent gout attacks and the presence of a tophus or tophi. ${ }^{13}$ EULAR guidelines are similar, recommending lifetime ULT for patients with recurrent gout attacks, chronic arthropathy, tophaceous deposits, nephrolithiasis, and/or radiographic changes associated with gout. ${ }^{11}$ Both ACR and EULAR recommend a xanthine oxidase inhibitor (XOI) as first-line treatment, namely, allopurinol or febuxostat. Probenecid is an alternative for patients who are intolerant of XOIs or for whom XOIs are contraindicated. The goal of ULT is to maintain serum urate levels below $6 \mathrm{mg} / \mathrm{dL} .{ }^{11,13}$

Despite the availability of ULT, many gout patients do not achieve target serum urate levels, in part due to poor adherence to therapy in clinical practice. ${ }^{14-17}$ In US studies of patients newly initiating ULT, $56 \%-64 \%$ of patients were classified as non-adherent (based on a medication possession ratio less than 0.80 ) in the 1 st year of treatment. ${ }^{15,17}$ There are multiple barriers to adherence; however, several studies in multiple therapeutic areas have demonstrated a positive link between treatment satisfaction and adherence to therapy. ${ }^{18,19}$ Treatment satisfaction is also positively correlated with HRQoL. ${ }^{19-21}$ Assessment of treatment satisfaction and HRQoL provides an opportunity to incorporate patient perspectives into clinical decision-making, which should ultimately improve the quality and value of health care. ${ }^{22}$

The Gout Impact Scale (GIS) is a validated disease-specific instrument that assesses the impact of gout on HRQoL. ${ }^{23}$ The GIS showed evidence of validity to assess disease-specific functioning and health in patients with gout and seems to capture different aspects than those represented in the Health Assessment Questionnaire and SF-36 in a real-world setting. ${ }^{24}$ The Treatment Satisfaction Questionnaire for Medication (TSQM) is a validated measure of patient satisfaction with medication. ${ }^{25}$ The TSQM has been used in studies of other rheumatic diseases ${ }^{26,27}$ but, to our knowledge, has not been evaluated in patients with gout. Comparing results of both HRQoL and treatment satisfaction instruments in clinical practice will help inform the selection of tools that provide meaningful insight to clinicians managing gout patients. The objective of this study was to assess and compare treatment satisfaction with the TSQM, gout-specific HRQoL with the GIS, and generic HRQoL with the SF-12v2 ${ }^{\circledR}$ Health Survey (SF-12) in gout patients in a real-world practice setting, including the assessment of the ability to discriminate between patients with and without a gout attack.

\section{Methods}

\section{Study overview}

This study was a cross-sectional mail survey of patients with self-reported gout who were taking febuxostat ${ }^{28}$ at the time of the survey. The study protocol received ethics approval and a waiver of authorization from the New England Institutional Review Board (NEIRB). Patients were invited to participate via a mailed invitation that included a consent form, health information release form, the survey and notification that a US\$25 incentive would be sent to patients who completed the survey. The survey was completed AugustNovember 2012.

\section{Patient identification}

The study population included commercial health plan members identified in the Optum Research Database which contains claims data for approximately 33.9 million patients with commercial insurance, from all US geographic census regions. ${ }^{29}$ Health plan members who met the following criteria were eligible to receive an invitation to participate in the study: 1) $\geq 1$ claim for febuxostat between April 26, 2012 and July 26, 2012; 2) age $\geq 18$ years as of January 1, 2012 (ie, year of febuxostat prescription); 3) valid provider and patient information; 4) current enrollment in the health plan; and 5) usable patient health information. Patients with a self-reported diagnosis of gout on the survey, who could read and understand English, and who completed the consent and health and information release forms, were included in the analysis. Patients were excluded if they reported a cancer diagnosis in the previous year, had never taken febuxostat, had discontinued febuxostat prior to completing the survey, or had initiated a ULT in addition to febuxostat.

\section{Survey}

Patients reported demographic (age, sex, and race), socioeconomic (highest education level, employment status, occupation, and occupational activities), and clinical (height, weight, and comorbidities) data. Gout status was assessed by asking patients if they had been diagnosed with gout and 
whether they were currently experiencing a gout attack. Medication data included ULT taken prior to febuxostat and concomitant medications to prevent gout attacks or flares. Febuxostat-related data included start and stop dates, dose strength and frequency, and discontinuation information, including any new ULT started.

Treatment satisfaction was assessed by the TSQM version II. ${ }^{30}$ Patients were asked on the TSQM to rate their level of satisfaction with the ULT they were currently taking for gout. The TSQM has eleven items, representing four domains (Table 1) scored from 0 to 100, with higher scores indicating better satisfaction.

HRQoL was assessed by the GIS ${ }^{23,31}$ and SF-12...$^{32,33}$ The GIS is a disease-specific HRQoL instrument that measures the impact of gout on HRQoL and contains 24 questions representing five scales (Table 1). Each scale has a score of 0-100 with higher scores indicating "worse condition/ impairment" or "greater gout impact". The SF-12 is a generic HRQoL instrument with eight scales and two summary scores: the physical component summary (PCS) and mental component summary (MCS). $T$ scores for PCS and MCS were calculated by transforming raw scores into norm-based scores to achieve the same mean (50) and standard deviation (SD, 10) of the 2009 US general population normative sample. ${ }^{32}$

\section{Statistical analyses}

The sample size was calculated to obtain a pre-specified level of precision for the mean TSQM global satisfaction score. A sample size of 219 is large enough to expect the width of a 95\% confidence interval around the mean TSQM global satisfaction score to be no more than 6, assuming a TSQM global satisfaction score SD of 22.6. ${ }^{25}$

The primary outcomes were treatment satisfaction (TSQM scores) and gout-specific HRQoL (GIS scores). Secondary analyses included assessment of the influence of current gout attack status on patient characteristics, TSQM, GIS, and SF-12 scores, correlations among TSQM, GIS, and SF-12 scales, and patient characteristics associated with TSQM domain scores. The influence of current gout attack status (yes/no) was tested by two-sample $t$-tests for continuous variables and chi-square test for categorical variables. Pearson correlation coefficients were calculated for the TSQM, GIS, and SF-12 scores. We categorized the strength of the correlation (based on the absolute values of $r$ ) as very weak $(r=0-0.19)$, weak $(r=0.20-0.39)$, moderate $(r=0.40-0.59)$, relatively strong $(r=0.60-0.79)$, and strong $(r=0.8-1){ }^{34}$

Characteristics of patients most satisfied with treatment were assessed by multivariate regression models,

Table I Concepts from the TSQM and example items from GIS

\begin{tabular}{|c|c|}
\hline \multirow{2}{*}{$\begin{array}{l}\text { TSQM domains } \\
\text { Effectiveness }\end{array}$} & Concepts \\
\hline & $\begin{array}{l}\text { - Prevent or treat the condition } \\
\text { - Relief of symptoms }\end{array}$ \\
\hline Side effects & $\begin{array}{l}\text { - Interference with physical functioning } \\
\text { - Interference with mental functioning } \\
\text { - Interference with mood or emotions }\end{array}$ \\
\hline Convenience & $\begin{array}{l}\text { - Ease of medication use } \\
\text { - Ease of planning } \\
\text { - Frequency of medication use }\end{array}$ \\
\hline Global satisfaction & - Global satisfaction \\
\hline $\begin{array}{l}\text { GIS scales } \\
\text { Impact of gout overall (three sca }\end{array}$ & Example items \\
\hline Gout concern overall & $\begin{array}{l}\text { - I am worried that I will have a gout attack within the next year } \\
\text { - I worry that I will not be able to continue to enjoy my leisure activities as a result of my gout }\end{array}$ \\
\hline Gout medication side effects & $\begin{array}{l}\text { - I am bothered by side effects from my gout medications } \\
\text { - I worry about long-term effects of gout medications }\end{array}$ \\
\hline Unmet gout treatment need & $\begin{array}{l}\text { - I have control over my gout } \\
\text { - My current medications do not work well to prevent gout attacks from happening }\end{array}$ \\
\hline \multicolumn{2}{|l|}{ During gout attack (two scales) } \\
\hline Gout concern during attack & $\begin{array}{l}\text { - It is difficult to plan ahead for events or activities because I may have a gout attack } \\
\text { - I am mad or angry when I experience a gout attack }\end{array}$ \\
\hline Well-being during attack & $\begin{array}{l}\text { - How much did your symptoms interfere with your normal work (including both work } \\
\text { outside the home and housework) } \\
\text { - How much did your symptoms interfere with your enjoyment of life? }\end{array}$ \\
\hline
\end{tabular}

Abbreviations: GIS, Gout Impact Scale; TSQM, Treatment Satisfaction Questionnaire for Medication. 
one for each TSQM domain. The dependent variable was the TSQM domain score and independent variables were age, race, ethnicity, sex, body mass index, employment status, occupation, occupational activities, highest level of education, febuxostat user status (new or continuing user; new users started febuxostat within 60 days of survey completion), febuxostat dose per tablet, satisfaction with febuxostat compared with prior ULT, concomitant nonULT medication(s) to prevent gout attack/flares, current gout attack status, count of comorbid conditions, and normative score of SF-12 bodily pain scale $(<47,47-53,>53)$. Pairwise correlations among independent variables were examined to assess orthogonality of study design. A leastsquares regression model was used for the TSQM domains of effectiveness, convenience, and global satisfaction using $F$-tests for significance of independent variables. Because nearly $80 \%$ of subjects had a side effect domain score equal to 100 (the maximum possible), a logistic regression model was used for the side effect domain score as a dichotomous variable ( 1 if side effect score equaled 100, 0 otherwise) and chi-square tests were used for significance of independent variables.

\section{Results \\ Patient identification}

There were 3,272 health plan enrollees with $\geq 1$ claim for febuxostat between April 26, 2012 and July 26, 2012 (Figure 1A). Of these enrollees, 1,206 (36.9\%) were survey eligible and were mailed a survey. There were 281 valid respondents for a survey response rate of $23.3 \%$ (Figure 1B). Twentyfour respondents $(8.5 \%)$ were excluded because, at the time of the survey, they had either discontinued febuxostat $(n=21)$ or were taking another ULT in addition to febuxostat $(n=3)$. The final analytic sample included 257 patients who were taking only febuxostat when they completed the survey.

\section{Patient characteristics}

Mean (SD) age was 54.9 (9.8) years and $87.4 \%$ of patients were men (Table 2). At the time of the survey, 29 patients (11.4\%) were experiencing a gout attack, and 56.4\% $(145 / 257)$ of patients reported that they were taking concomitant gout medication (a non-ULT) to prevent gout flares/attacks. Only seven patients (3.2\%) were classified as new users. Comparing patients with a gout attack to patients without a gout attack, patients with an attack had a lesser

A

Health plan enrollees with $\geq 1$ claim for febuxostat between April 26, 2012 and July 26, 2012 $\mathrm{N}=3,272$

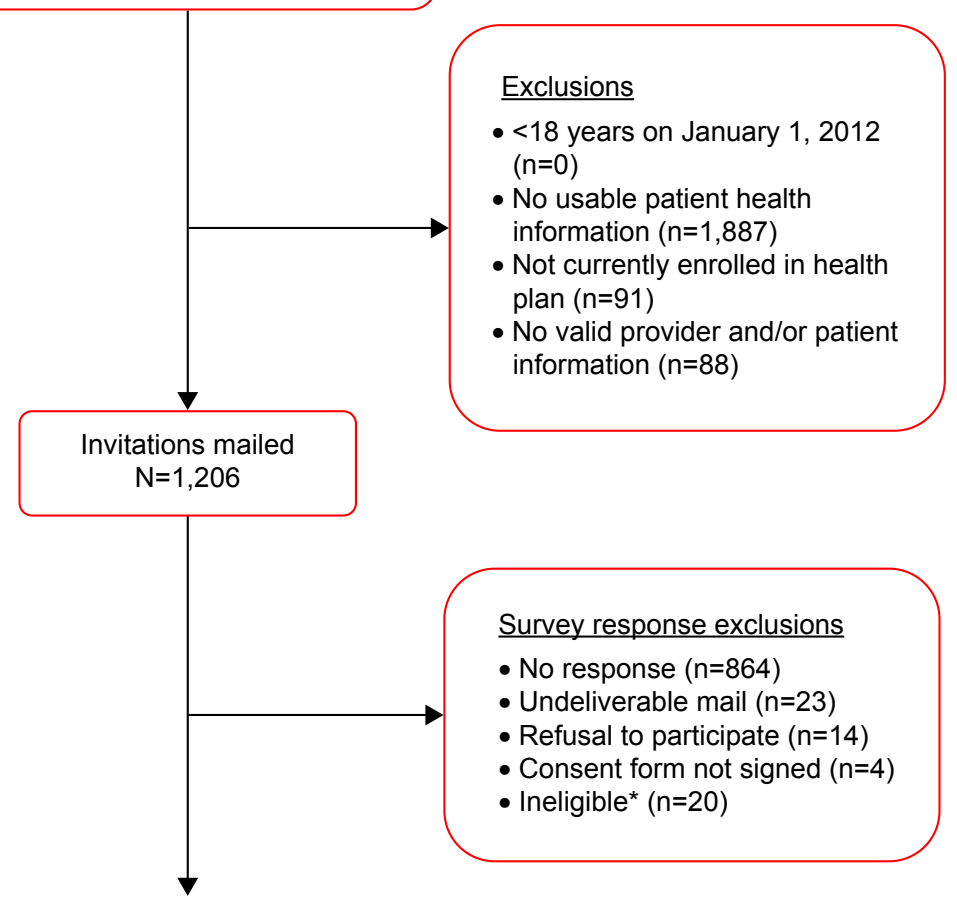




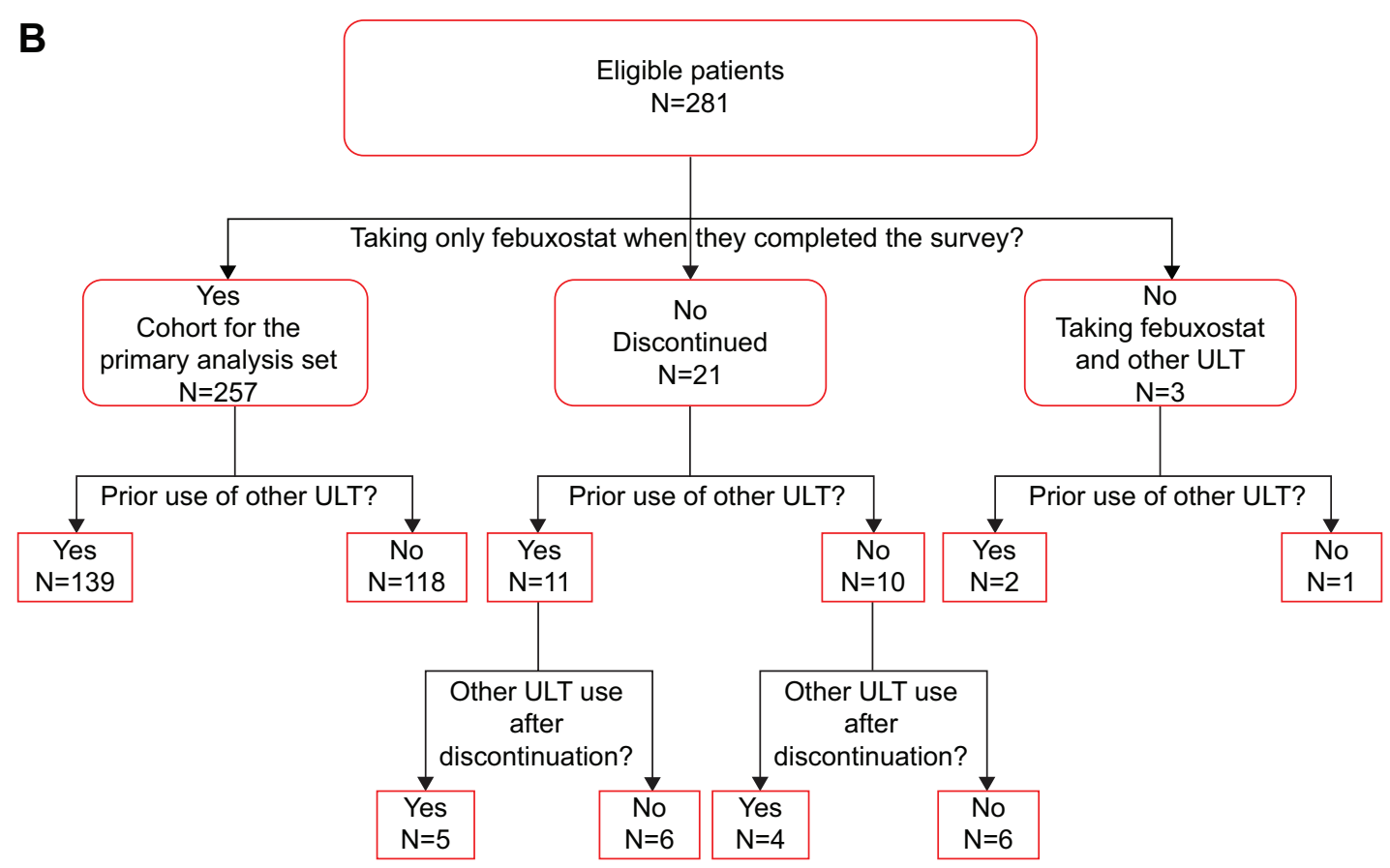

Figure I Sample identification and survey response $(\mathbf{A})$ and study participants (B).

Notes: *No longer enrolled (I), no gout (5), cancer in past year (9), never taken febuxostat (2) language barrier (I), deceased (2).

Abbreviation: ULT, urate-lowering therapy.

Table 2 Patient characteristics for all patients and by gout attack status

\begin{tabular}{|c|c|c|c|c|c|c|c|}
\hline & \multirow{2}{*}{\multicolumn{2}{|c|}{$\begin{array}{l}\text { All patients } \\
(n=257)\end{array}$}} & \multicolumn{5}{|c|}{ Current gout attack? $(n=255)^{b}$} \\
\hline & & & \multicolumn{2}{|c|}{ Yes $(n=29)$} & \multicolumn{2}{|c|}{ No $(n=226)$} & \multirow{2}{*}{$\begin{array}{l}P \text {-value } \\
\text { (yes vs no) }\end{array}$} \\
\hline & n & & n & & $\bar{n}$ & & \\
\hline Age (years), mean (SD) & 253 & $54.9(9.8)$ & 29 & $55.5(11.1)$ & 222 & $54.8(9.7)$ & 0.722 \\
\hline \multicolumn{8}{|l|}{ Age group, $\mathrm{n}(\%)$} \\
\hline $18-49$ years & & $68(26.9)$ & & $8(27.6)$ & & $60(27.0)$ & 0.949 \\
\hline $50-64$ years & & $149(58.9)$ & & $16(55.2)$ & & $|3|(59.0)$ & 0.693 \\
\hline$\geq 65$ years & & $36(14.2)$ & & $5(17.2)$ & & $31(14.0)$ & 0.636 \\
\hline Male, n (\%) & 254 & $222(87.4)$ & 29 & $21(72.4)$ & 223 & $199(89.2)$ & 0.010 \\
\hline Race, n (\%) & 252 & & 29 & & 221 & & \\
\hline White & & $228(90.5)$ & & $25(86.2)$ & & $202(9 \mid .4)$ & 0.363 \\
\hline Black & & $18(7.1)$ & & $3(10.3)$ & & $14(6.3)$ & 0.420 \\
\hline Asian & & $4(1.6)$ & & 0 & & $3(1.4)$ & 0.528 \\
\hline American Indian & & $3(1.2)$ & & I (3.4) & & $3(1.4)$ & 0.399 \\
\hline Other & & $3(1.2)$ & & 0 & & $3(1.4)$ & 0.528 \\
\hline Employment, n (\%) & 257 & & 29 & & 226 & & \\
\hline Full-time & & $199(77.4)$ & & $20(69.0)$ & & I 78 (78.8) & 0.233 \\
\hline Part-time & & $13(5.1)$ & & $2(6.9)$ & & II (4.9) & 0.640 \\
\hline Retired & & $29(11.3)$ & & $2(6.9)$ & & $26(11.5)$ & 0.455 \\
\hline Homemaker, unemployed, other & & $19(7.4)$ & & $5(I 7.2)$ & & $14(6.2)$ & 0.033 \\
\hline Occupational activities, n (\%) & 243 & & 25 & & 216 & & \\
\hline Mostly sitting, little walking & & $105(43.2)$ & & $9(36.0)$ & & $96(44.4)$ & 0.420 \\
\hline Stand, walk, little lifting & & $94(38.7)$ & & $8(32.0)$ & & $86(39.8)$ & 0.448 \\
\hline Light lifting, climbing stairs & & $27(I I . I)$ & & $6(24.0)$ & & $20(9.3)$ & 0.025 \\
\hline Heavy work or carrying & & $17(7.0)$ & & $2(8.0)$ & & $14(6.5)$ & 0.773 \\
\hline Education, $\mathrm{n}(\%)$ & 253 & & 29 & & 222 & & \\
\hline High school or less & & $41(16.2)$ & & $4(\mid 3.8)$ & & $36(16.2)$ & 0.737 \\
\hline$<4$ years college & & $86(34.0)$ & & $10(34.5)$ & & $76(34.2)$ & 0.979 \\
\hline$\geq 4$ year degree & & $126(49.8)$ & & 15 (5।.7) & & $110(49.5)$ & 0.826 \\
\hline
\end{tabular}


Table 2 (Continued)

\begin{tabular}{|c|c|c|c|c|c|c|c|}
\hline & \multirow{2}{*}{\multicolumn{2}{|c|}{$\begin{array}{l}\text { All patients }{ }^{a} \\
(n=257)\end{array}$}} & \multicolumn{5}{|c|}{ Current gout attack? $(\mathrm{n}=255)^{\mathrm{b}}$} \\
\hline & & & \multicolumn{2}{|c|}{ Yes $(n=29)$} & \multicolumn{2}{|c|}{ No $(n=226)$} & \multirow{2}{*}{$\begin{array}{l}\begin{array}{l}P \text {-value } \\
\text { (yes vs no) }\end{array}\end{array}$} \\
\hline & $\bar{n}$ & & $\mathbf{n}$ & & $\bar{n}$ & & \\
\hline Febuxostat use status, $\mathrm{n}(\%)$ & 218 & & 26 & & 190 & & \\
\hline New users $(\leq 60 \text { days) })^{\mathrm{e}}$ & & $7(3.2)$ & & $3(11.5)$ & & $4(2.1)$ & 0.011 \\
\hline Continuing users & & $211(96.8)$ & & $23(88.5)$ & & $186(97.9)$ & 0.011 \\
\hline Concomitant gout medication, $\mathrm{n}(\%)^{\mathrm{d}}$ & 257 & $145(56.4)$ & 29 & $19(65.5)$ & 226 & $124(54.9)$ & 0.277 \\
\hline Colchicine & & $128(49.8)$ & & $16(55.2)$ & & $112(49.6)$ & 0.569 \\
\hline NSAID & & $31(12.1)$ & & $7(24.2)$ & & $23(10.2)$ & 0.028 \\
\hline Corticosteroid & & $6(2.3)$ & & 0 & & $6(2.7)$ & 0.375 \\
\hline Opioid & & $\mathrm{I}(0.4)$ & & 0 & & $\mathrm{I}(0.4)$ & 0.720 \\
\hline Five most common comorbidities, $\mathrm{n}(\%)^{\mathrm{d}}$ & 254 & & 29 & & 223 & & \\
\hline High blood pressure & & $160(63.0)$ & & $20(69.0)$ & & $138(61.9)$ & 0.458 \\
\hline Back pain & & $60(23.6)$ & & $7(24.1)$ & & $52(23.3)$ & 0.922 \\
\hline Diabetes & & $55(21.7)$ & & $7(24.1)$ & & $47(21.1)$ & 0.705 \\
\hline Kidney disease & & $29(11.4)$ & & $4(13.8)$ & & $24(10.8)$ & 0.625 \\
\hline Osteoarthritis, degenerative arthritis & & $24(9.5)$ & & $3(10.3)$ & & $21(9.4)$ & 0.873 \\
\hline Number of non-gout conditions, $\mathrm{n}(\%)$ & 254 & & 29 & & 223 & & \\
\hline 0 & & $46(18.1)$ & & $2(6.9)$ & & $44(19.7)$ & 0.092 \\
\hline $1-2$ & & $150(59.1)$ & & $21(72.4)$ & & $129(57.8)$ & 0.133 \\
\hline $3-4$ & & $42(16.5)$ & & $4(13.8)$ & & $36(16.1)$ & 0.745 \\
\hline $5+$ & & $16(6.3)$ & & $2(6.9)$ & & $14(6.3)$ & 0.898 \\
\hline Body mass index, mean (SD) & 254 & $32.4(6.9)$ & 29 & $32.2(7.6)$ & 223 & $32.5(6.9)$ & 0.834 \\
\hline Body mass index group $\left(\mathrm{kg} / \mathrm{m}^{2}\right), \mathrm{n}(\%)$ & & & & & & & \\
\hline Underweight $(<18.5)$ & & $2(0.8)$ & & I (3.4) & & $\mathrm{I}(0.4)$ & 0.087 \\
\hline Normal (18.5-24.9) & & $16(6.3)$ & & $2(6.9)$ & & $14(6.3)$ & 0.898 \\
\hline Overweight (25-29.9) & & $93(36.6)$ & & $9(31.0)$ & & $83(37.2)$ & 0.515 \\
\hline Obese $(30-34.9)$ & & $72(28.4)$ & & $9(31.0)$ & & $62(27.8)$ & 0.716 \\
\hline Severely obese $(\geq 35)$ & & $71(28.0)$ & & $8(27.6)$ & & $63(28.3)$ & 0.940 \\
\hline
\end{tabular}

Notes: a $\mathrm{N}<257$ for some characteristics due to missing values on survey; ${ }^{\mathrm{b}} \mathrm{Of}$ the 257 patients, 255 had data for current gout attack; ' $\mathrm{D}$ ifference between gout attack and no gout attack assessed by two-sample $t$-test for continuous variables and chi-square test for categorical variables; ${ }^{\mathrm{d} P a t i e n t s}$ could report more than category; and ${ }^{\mathrm{P} P r e s c r i p t i o n}$ for febuxostat $\leq 60$ days before survey completion.

Abbreviations: SD, standard deviation; NSAID, non-steroidal anti-inflammatory drug.

proportion of males ( $72.4 \%$ vs $89.2 \%$ ), were a homemaker, unemployed, or other (17.2\% vs 6.2\%), had an occupational action of lifting or climbing stairs often $(24.0 \%$ vs $9.3 \%)$, were new users (11.5\% vs $2.1 \%$ ), and were taking concomitant non-steroidal anti-inflammatory drugs $(24.1 \%$ vs 10.2\%) (Table 2).

\section{Treatment patterns}

Mean (SD) and median daily doses were $54.5 \mathrm{mg}$ (19.3 mg) and $40 \mathrm{mg}$, respectively. Mean time between initiating febuxostat and completing the survey was 499 days (range: 6-1,345 days). Of the 21 patients who had discontinued febuxostat at the time of the survey, nine switched to a new ULT: allopurinol (seven patients), probenecid (one patient), and unknown ULT (one patient) (data not shown). Multiple reasons for discontinuation could be provided and these included cost (eight patients), side effects (five patients), symptom resolution (four patients), and ineffective medication (four patients).

\section{Treatment satisfaction}

Mean TSQM scores ranged from 75.4 (effectiveness) to 94.7 (side effects) with higher scores indicating better satisfaction (Table 3). Compared with patients with no current attack, patients with current attack had worse mean scores for all domains except convenience, with mean differences of -20.6 for effectiveness, -10.6 for side effects, -12.1 for global satisfaction (all $P<0.05$ ), and -6.1 for convenience (NS).

In the multivariate analysis of patient characteristics associated with TSQM domain scores, patients without gout attack had higher global satisfaction, effectiveness, and side effect scores than patients with gout attack. Global satisfaction scores were higher for patients with less bodily pain on the SF-12 than patients with average bodily pain. Older patients were more likely to have the best possible side effect of score (100). Pairwise correlations among independent variables revealed only very weak/weak relationships. 
Table 3 Mean TSQM, GIS, and SF-I2 scores for all patients and by gout attack status

\begin{tabular}{|c|c|c|c|c|c|}
\hline & \multirow{2}{*}{$\begin{array}{l}\text { All patients } \\
(n=255)^{a}\end{array}$} & \multicolumn{4}{|c|}{ Current gout attack? $(n=255)^{a}$} \\
\hline & & \multirow{2}{*}{$\begin{array}{l}\text { Yes }(n=29) \\
\overline{\text { Mean (SD) }}\end{array}$} & \multirow{2}{*}{$\begin{array}{l}\text { No }(n=226) \\
\text { Mean (SD) }\end{array}$} & \multirow{2}{*}{$\begin{array}{l}\text { Difference: } \\
\text { attack vs no attack } \\
\text { Mean }(95 \% \mathrm{Cl})\end{array}$} & \multirow[t]{2}{*}{$\begin{array}{l}\text { P-value } \\
\text { (yes vs no) }\end{array}$} \\
\hline & $\overline{\text { Mean (SD) }}$ & & & & \\
\hline \multicolumn{6}{|l|}{ TSQM vll domain scores: $0-100^{\circ}$} \\
\hline Effectiveness & $75.4(26.1)$ & $57.2(26.0)$ & $77.7(25.2)$ & $-20.6(-30.4,-10.7)$ & $<0.001$ \\
\hline Side effects & $94.7(14.1)$ & $85.3(26.3)$ & $95.9(11.1)$ & $-10.6(-20.7,-0.5)$ & 0.041 \\
\hline Convenience & $83.4(15.8)$ & $78.0(19.8)$ & $84.1(15.2)$ & $-6.1(-13.9,1.7)$ & 0.121 \\
\hline Global satisfaction & $80.0(19.4)$ & $69.3(21.0)$ & $81.4(18.8)$ & $-12.1(-19.5,-4.7)$ & 0.001 \\
\hline \multicolumn{6}{|l|}{ Gout Impact Scale: $0-100^{d}$} \\
\hline Gout overall concern & $47.1(29.5)$ & $74.1(29.9)$ & $43.7(27.6)$ & $30.5(19.6,41.3)$ & $<0.001$ \\
\hline Gout medication side effects & $43.6(23.7)$ & $56.5(26.4)$ & $41.9(22.9)$ & $14.6(5.5,23.6)$ & 0.002 \\
\hline Unmet gout treatment needs & $28.2(21.4)$ & $48.3(26.2)$ & $25.6(19.3)$ & $22.7(12.4,32.9)$ & $<0.001$ \\
\hline Gout concern during attack & $47.2(24.2)$ & $57.4(27.0)$ & $45.9(23.5)$ & II.5 $(2.2,20.8)$ & 0.016 \\
\hline Well-being during attack & $54.7(25.0)$ & $61.7(26.2)$ & $53.8(24.8)$ & $7.9(-1.8,17.6)$ & 0.110 \\
\hline \multicolumn{6}{|l|}{ SF-I 2 scores $^{e}$} \\
\hline Physical component summary & $49.4(9.5)$ & $43.5(10.2)$ & $50.1(9.1)$ & $-6.6(-10.2,-3.0)$ & $<0.001$ \\
\hline Mental component summary & $52.9(7.9)$ & $50.3(9.9)$ & $53.2(7.5)$ & $-2.9(-6.8,1.0)$ & 0.137 \\
\hline
\end{tabular}

Notes: ${ }^{a}$ Of the 257 patients, 255 had data for current gout attack; 'Difference between gout attack and no gout attack assessed by two-sample $t$-test; 'Higher scores indicate better satisfaction; ${ }^{~}$ Higher scores indicate worse condition or greater gout impairment; and ${ }^{\mathrm{e}} T$ scores between 47 and 53 are within average range; those less than 47 indicate impaired functioning. The lowest and highest possible $T$ scores, respectively, are: physical component summary, II.07 and 71.78 ; mental component summary, 11.29 and $68.15 .^{32}$

Abbreviations: Cl, confidence interval; SD, standard deviation; TSQM vll, Treatment Satisfaction Questionnaire for Medication version II; GIS, Gout Impact Scale; SF-I2, SF-I 2 $2{ }^{\circledR}$ Health Survey.

\section{Health-related quality of life}

Mean GIS summary scores ranged from 28.2 (unmet gout treatment needs) to 54.7 (well-being during attack) with higher scores indicating worse condition/impairment or greater gout impact (Table 3). Patients with a current gout attack had significantly higher (ie, worse) mean scores than patients without a current gout attack on all scales except well-being during attack. Differences in mean scores between current and no current attack were: 30.5 , gout overall concern; 14.6, gout medication side effects; 22.7, unmet gout treatment needs; 11.5 , gout concern during attack (all $P<0.05$ ); and 7.9, well-being during attack. Mean SF-12 scores for all patients ( $\mathrm{n}=255$ ) were 49.4 for the PCS and 52.9 for the MCS (Table 3). Patients with a current gout attack had lower mean scores on the PCS $(-6.6, P<0.001)$ and MCS $(-2.9)$ but the difference in MCS score was not significant.

\section{Correlations among instruments}

There were weak to moderate correlations between scores on different instruments scales and moderate to relatively strong correlations between certain scale scores within the same instrument (Table 4). These included moderate correlations between GIS gout medication side effect and TSQM side effects (-0.46), the GIS unmet gout treatment needs and TSQM effectiveness ( -0.40$)$, and the GIS unmet gout treatment needs and TSQM global satisfaction $(-0.50)$.
Correlations between the TSQM and SF-12 scales and between the GIS and SF-12 scales were all very weak or weak. Correlations between TSQM global satisfaction and both effectiveness and convenience were relatively strong (0.67 and 0.76 ). GIS gout overall concern and all other GIS scales (except well-being during attack, 0.26) were moderately correlated (0.52-0.59), as were GIS gout concern during attack and gout medication side effects $(0.53)$. The correlation between GIS gout concern during attack and well-being during attack was relatively strong (0.61).

\section{Discussion}

To our knowledge, this is the first study in gout patients to assess treatment satisfaction with the TSQM and to compare the TSQM with the GIS. Both instruments were effective in discriminating the effect of gout attack status with significantly worse scores on three of four TSQM scales and four of five GIS scales for patients currently experiencing a gout attack compared with patients who were not experiencing a gout attack. Similarly, the patient characteristic most predictive of lower treatment satisfaction was the presence of a current gout attack. There were several moderate correlations between GIS and TSQM scales that supported the relationship between gout treatment satisfaction and gout-related quality of life.

Gout-specific HRQoL as measured by the GIS showed that patients did have some concern about their overall gout, 


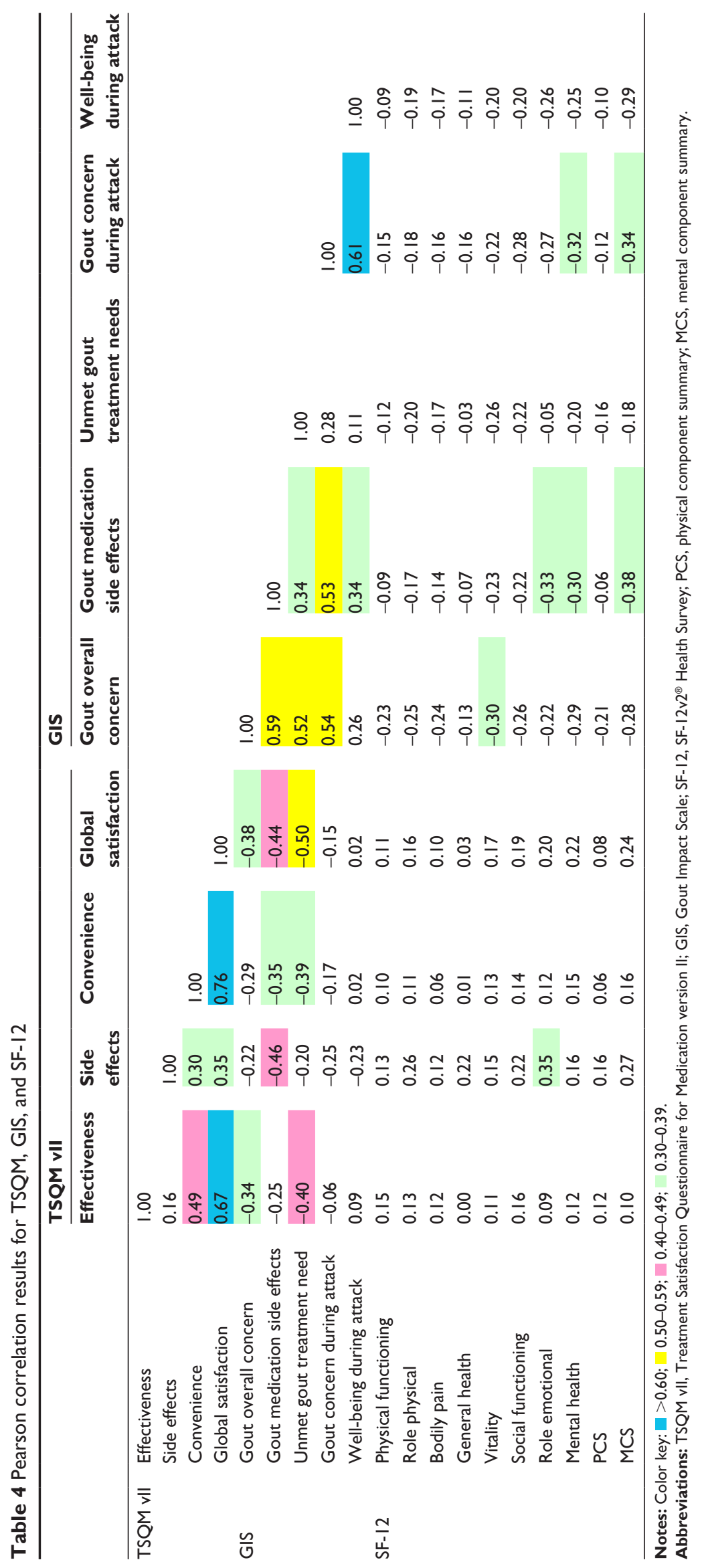


gout medication side effects, and concern about gout and wellbeing during their gout attack. However, patients indicated that their treatment was adequate as shown by lower scores on the unmet gout treatment need score. Mean GIS scores across all patients ranged from 28.2 to 54.7 and were lower (indicating less gout impairment) than mean GIS scores observed by Hirsch et al with mean differences of 1.1 (well-being during attack) to 16.0 points (gout concern overall). ${ }^{35}$ Our study was specific to ULT medication for gout prevention and all respondents were required to have a febuxostat prescription; $7.4 \%$ of patients in the earlier study were not taking any gout medication and, of the $92.6 \%$ of patients who were on therapy, the medication could have been for acute treatment of gout flares and/or gout prevention. Patients in the earlier study were also older on average than our patients (62.2 vs 54.9 years). However, both studies found a trend toward worsening GIS scores (except for well-being during attack) with increasing attack frequency ${ }^{35}$ or current attack status (our study). The estimated minimally important differences for the GIS scales range from 5.2 to 7.6 points, ${ }^{36}$ and we observed worse scores for patients currently experiencing a gout attack vs those not experiencing an attack with mean differences ranging from 7.9 to 30.5 points. These results, coupled with recent studies of the GIS, ${ }^{35,36}$ support its feasibility (patients were able to complete the GIS themselves) and validity (correlations with other instruments and differences between current gout attack and no current gout attack) for assessing HRQoL in gout.

Generic HRQoL scores as measured by the SF-12 were within the average range of the normative US sample (47-53): mean $T$ scores were 49.4 for the PCS and 52.9 for the MCS. Our mean scores are higher than previously reported SF-36 scores for gout patients on the PCS (37.9) and MCS (48.5), with the largest mean difference evident in PCS scores. ${ }^{5}$ However, only approximately two-thirds of patients in the earlier study reported taking any current gout medication (allopurinol and/or colchicine) and they were older on average than our patients (62.2 years vs 54.9 years), which likely contributed to lower scores. Almost all patients (98.7\%) in our study were continuous users of febuxostat, having taken it on average for 499 days. Our PCS and MCS results are consistent with a study that examined mean SF-36 scores of patients closer in age (57.1 years) to our patients and who had been taking ULT therapy for 1 year (mean PCS, 48.5; mean MCS, 54.2). ${ }^{37}$ The PCS and MCS were able to discriminate patients with gout attack vs no attack with mean differences on the PCS (-6.6) and MCS (-2.9) that are at or above the clinically meaningful difference of 3 points. ${ }^{32}$ The impact of gout attack was most pronounced for the PCS which is consistent with a previous study which observed larger decrements in the PCS than the MCS with increasing frequency of gout flares..$^{38}$ Only weak correlations were found between the SF-12 and the TSQM and GIS.

The TSQM discriminated gout attack status, with patients who were currently experiencing a gout attack registering significantly lower mean scores on all scales except convenience. Differences in convenience scores by gout attack status would not be expected since the indication for febuxostat is once daily dosing, regardless of attack status. The findings that patients experiencing an attack scored higher on medication side effects could imply that patients thought that an attack could be a side effect of medication or that patients with side effects are more likely to stop their medication and therefore experience an attack. There were several moderate correlations between the TSQM and GIS scales, supporting the relationship between treatment satisfaction and HRQoL. The moderate correlations between the GIS unmet gout treatment needs and TSQM global satisfaction $(-0.50)$ and effectiveness $(-0.40)$ scales suggest that both measures captured the degree to which patients believed that their medication was beneficial for their gout treatment. The correlation between the side effect scales of the GIS and TSQM was also moderate $(-0.46)$, indicating consistent findings from both instruments.

Patient-reported outcomes are an important consideration in the clinical management of patients with gout. While clinical assessment will be the basis for diagnosis, patient-reported outcomes provide important inputs for understanding satisfaction with treatment and the impact of gout on daily living, and for tracking response to treatment. Measuring treatment satisfaction is also a solid first step in shared decision-making to promote better adherence to therapy and, ultimately, improved clinical and quality-of-life outcomes. The TSQM has eleven items vs the 24-item GIS and may offer greater convenience in busy clinical practice settings. However, the GIS captures additional information on gout concern overall and status during a gout attack, both important considerations for disease management. Nevertheless, additional research is needed to support routine adoption of these measures in clinical practice. The minimally important difference for the TSQM scales needs to be determined and Outcome Measures in Rheumatology (OMERACT) has not endorsed two scales of the GIS: gout concern overall and unmet gout treatment needs. ${ }^{39}$ Our results provide further evidence of the performance of these measures in a real-world setting to advance future research and, coupled with previous research, ${ }^{35,36}$ provide further psychometric properties to support GIS as an appropriate measure. 
There are several limitations to this study which should be considered. This was a cross-sectional survey of a convenience sample of patients and the self-reported results are subject to recall bias. Almost all patients (96.8\%) were taking febuxostat for at least 60 days, which may bias the results in favor of patients satisfied with treatment. The study also lacked a formal comparator group of patients taking a ULT other than febuxostat, so satisfaction with febuxostat cannot be compared to another benchmark. Finally, our results are based on commercial health plan enrollees and $82 \%$ were employed, so employed individuals are disproportionately represented.

Both the GIS and TSQM were complementary in evaluating the impact of disease and treatment satisfaction in patients treated with febuxostat in both acute and chronic gout. The SF-12 was able to discriminate acute attack vs not, but only weakly associated with treatment satisfaction and diseasespecific HRQOL. Current gout attack was the strongest predictor of patient satisfaction. Correlations between GIS and TSQM scales supported the relationship between treatment satisfaction and gout-specific quality of life.

\section{Acknowledgments}

The authors would like to thank Sarah Peirce-Sandner (Optum, Eden Prairie, MN, USA), who provided medical writing support and was funded by Takeda Pharmaceuticals International. The authors would also like to thank the individuals at Optum who were involved in the study at various stages: Margaret Good, Yvette Grenier, Fang Liu, Jeffrey McPheeters, Angela Watkins, Julia Xeroteres, and Bryan Zachau.

\section{Disclosure}

PP Khanna has served as a consultant to Takeda Pharmaceuticals International, Inc. A Shiozawa is an employee of Takeda Pharmaceuticals International, Inc., febuxostat is a product of Takeda. V Walker, T Bancroft, and B Essoi are employees of OptumInsight who were paid consultants to Takeda Pharmaceuticals International, Inc. in connection with this study. D Khanna has served as a consultant to AstraZeneca and Takeda Pharmaceuticals International, Inc. KS Akhras was employed by Takeda Pharmaceuticals International, Inc. at the time the research was conducted.

\section{References}

1. Tausche AK, Jansen TL, Schroder HE, Bornstein SR, Aringer M, MullerLadner U. Gout - current diagnosis and treatment. Dtsch Arztebl Int. 2009;106(34-35):549-555.

2. Terkeltaub R. Update on gout: new therapeutic strategies and options. Nat Rev Rheumatol. 2010;6(1):30-38.

3. Neogi T. Clinical practice. Gout. N Engl J Med. 2011;364(5):443-452.
4. Becker MA, Schumacher HR, Benjamin KL, et al. Quality of life and disability in patients with treatment-failure gout. J Rheumatol. 2009;36(5): 1041-1048.

5. Lee SJ, Hirsch JD, Terkeltaub R, et al. Perceptions of disease and health-related quality of life among patients with gout. Rheumatology (Oxford). 2009;48(5):582-586.

6. Roddy E, Zhang W, Doherty M. Is gout associated with reduced quality of life? A case-control study. Rheumatology (Oxford). 2007;46(9): 1441-1444.

7. Chandratre P, Roddy E, Clarson L, Richardson J, Hider SL, Mallen CD. Health-related quality of life in gout: a systematic review. Rheumatology (Oxford). 2013;52(11):2031-2040.

8. Wu EQ, Forsythe A, Guerin A, Yu AP, Latremouille-Viau D, Tsaneva M. Comorbidity burden, healthcare resource utilization, and costs in chronic gout patients refractory to conventional urate-lowering therapy. Am J Ther. 2012;19(6):e157-e166.

9. Wu EQ, Patel PA, Yu AP, et al. Disease-related and all-cause health care costs of elderly patients with gout. J Manag Care Pharm. 2008;14(2): 164-175.

10. Zhu Y, Pandya BJ, Choi HK. Prevalence of gout and hyperuricemia in the US general population: the National Health and Nutrition Examination Survey 2007-2008. Arthritis Rheum. 2011;63(10):3136-3141.

11. Hamburger M, Baraf HS, Adamson TC 3rd, et al. 2011 Recommendations for the diagnosis and management of gout and hyperuricemia. Postgrad Med. 2011;123(6 Suppl 1):3-36.

12. Khanna D, Khanna PP, Fitzgerald JD, et al. 2012 American College of Rheumatology guidelines for management of gout. Part 2: therapy and antiinflammatory prophylaxis of acute gouty arthritis. Arthritis Care Res (Hoboken). 2012;64(10):1447-1461.

13. Khanna D, Fitzgerald JD, Khanna PP, et al. 2012 American College of Rheumatology guidelines for management of gout. Part 1: systematic nonpharmacologic and pharmacologic therapeutic approaches to hyperuricemia. Arthritis Care Res (Hoboken). 2012;64(10): 1431-1446.

14. Harrold LR, Andrade SE, Briesacher B, et al. The dynamics of chronic gout treatment: medication gaps and return to therapy. Am J Med. 2010; 123(1):54-59.

15. Harrold LR, Andrade SE, Briesacher BA, et al. Adherence with uratelowering therapies for the treatment of gout. Arthritis Res Ther. 2009; 11(2):R46.

16. Riedel AA, Nelson M, Joseph-Ridge N, Wallace K, MacDonald P, Becker M. Compliance with allopurinol therapy among managed care enrollees with gout: a retrospective analysis of administrative claims. J Rheumatol. 2004;31(8):1575-1581.

17. Solomon DH, Avorn J, Levin R, Brookhart MA. Uric acid lowering therapy: prescribing patterns in a large cohort of older adults. Ann Rheum Dis. 2008;67(5):609-613.

18. Barbosa CD, Balp MM, Kulich K, Germain N, Rofail D. A literature review to explore the link between treatment satisfaction and adherence, compliance, and persistence. Patient Prefer Adherence. 2012;6: 39-48.

19. Delestras S, Roustit M, Bedouch P, et al. Comparison between two generic questionnaires to assess satisfaction with medication in chronic diseases. PLoS One. 2013;8(2):e56247.

20. Biderman A, Noff E, Harris SB, Friedman N, Levy A. Treatment satisfaction of diabetic patients: what are the contributing factors? Fam Pract. 2009;26(2):102-108.

21. Nett RB, Tiseo PJ, Almas M, Sikes CR. Patient satisfaction with eletriptan in the acute treatment of migraine in primary care. Int J Clin Pract. 2007;61(10):1677-1685.

22. American Medical Association. Getting the most for our health care dollars. Available from: http://www.ama-assn.org/resources/doc/ health-care-costs/prevention-and-wellness.pdf. Accessed September 11, 2013.

23. Hirsch JD, Lee SJ, Terkeltaub R, et al. Evaluation of an instrument assessing influence of Gout on health-related quality of life. J Rheumatol. 2008;35(12):2406-2414. 
24. Spaetgens B, van der Linden S, Boonen A. The Gout Assessment Questionnaire 2.0: cross-cultural translation into Dutch, aspects of validity and linking to the International Classification of Functioning, Disability and Health. Rheumatology (Oxford). 2014;53(4): 678-685.

25. Atkinson MJ, Sinha A, Hass SL, et al. Validation of a general measure of treatment satisfaction, the Treatment Satisfaction Questionnaire for Medication (TSQM), using a national panel study of chronic disease. Health Qual Life Outcomes. 2004;2:12.

26. Jobanputra P, Maggs F, Deeming A, et al. A randomised efficacy and discontinuation study of etanercept versus adalimumab (RED SEA) for rheumatoid arthritis: a pragmatic, unblinded, non-inferiority study of first TNF inhibitor use: outcomes over 2 years. BMJ Open. 2012;2(6):e01395.

27. Lin HY, Cheng TT, Wang JH, et al. Etoricoxib improves pain, function and quality of life: results of a real-world effectiveness trial. Int $J$ Rheum Dis. 2010;13(2):144-150.

28. Takeda Pharmaceuticals America I. Uloric (febuxostat). Prescribing information. 2013. Available from: http://www.uloric.com/. Accessed September 17, 2013.

29. Census Regions and Divisions of the United States. US Census Bureau. http://www2.census.gov/geo/pdfs/maps-data/maps/reference/us_regdiv.pdf. Accessed May 22, 2015.

30. Atkinson MJ, Kumar R, Cappelleri JC, Hass SL. Hierarchical construct validity of the Treatment Satisfaction Questionnaire for Medication (TSQM version II) among outpatient pharmacy consumers. Value Health. 2005;8(Suppl 1):S9-S24.

31. Colwell HH, Hunt BJ, Pasta DJ, Palo WA, Mathias SD, Joseph-Ridge N. Gout Assessment Questionnaire: initial results of reliability, validity and responsiveness. Int J Clin Pract. 2006;60(10):1210-1217.
32. Maruish ME, editor. User's Manual for the SF-12v2 Health Survey. 3rd ed. Lincoln (RI): QualityMetric Inc.; 2012.

33. Ware J Jr, Kosinski M, Keller SD. A 12-Item Short-Form Health Survey: construction of scales and preliminary tests of reliability and validity. Med Care. 1996;34(3):220-233.

34. BMJ. Correlation and regression. Available from: http://www.bmj. com/about-bmj/resources-readers/publications/statistics-square-one/11correlation-and-regression. Accessed December 6, 2013.

35. Hirsch JD, Terkeltaub R, Khanna D, et al. Gout disease-specific quality of life and the association with gout characteristics. Patient Relat Outcome Meas. 2010;2010:1-8.

36. Khanna D, Sarkin AJ, Khanna PP, et al. Minimally important differences of the Gout Impact Scale in a randomized controlled trial. Rheumatology (Oxford). 2011;50(7):1331-1336.

37. Khanna PP, Perez-Ruiz F, Maranian P, Khanna D. Long-term therapy for chronic gout results in clinically important improvements in the health-related quality of life: short form-36 is responsive to change in chronic gout. Rheumatology (Oxford). 2011;50(4):740-745.

38. Khanna PP, Nuki G, Bardin T, et al. Tophi and frequent gout flares are associated with impairments to quality of life, productivity, and increased healthcare resource use: results from a cross-sectional survey. Health Qual Life Outcomes. 2012;10:117.

39. Singh JA, Taylor WJ, Simon LS, et al. Patient-reported outcomes in chronic gout: a report from OMERACT 10. J Rheumatol. 2011;38(7) 1452-1457.
Patient Preference and Adherence

\section{Publish your work in this journal}

Patient Preference and Adherence is an international, peer-reviewed, open access journal that focuses on the growing importance of patient preference and adherence throughout the therapeutic continuum. Patient satisfaction, acceptability, quality of life, compliance, persistence and their role in developing new therapeutic modalities and compounds to optimize

\section{Dovepress}

clinical outcomes for existing disease states are major areas of interest for the journal. This journal has been accepted for indexing on PubMed Central. The manuscript management system is completely online and includes a very quick and fair peer-review system, which is all easy to use. Visit http://www. dovepress.com/testimonials.php to read real quotes from published authors. 